\title{
THE INTERACTION BETWEEN SHOCK WAVES AND SOLID SPHERES ARRAYS IN A SHOCK TUBE *
}

\author{
SHI Honghui (施红辉) ${ }^{1,2, \dagger}$ Kazuki YAMAMURA ${ }^{3}$ \\ ${ }^{1}$ (State Key Laboratory of Nonlinear Mechanics, Institute of Mechanics, Chinese Academy of Sciences, \\ Beijing 100080, China) \\ ${ }^{2}$ (College of Mechanical and Automatic Control Engineering, Zhejiang University of Sciences, \\ Xiasha Higher Education Zone, Hangzhou 310018, China) \\ ${ }^{3}$ (Department of Mechanical Engineering, Nagoya Institute of Technology, Gokiso-cho, \\ Showa-ku, Nagoya 466-8555, Japan)
}

\begin{abstract}
When a shock wave interacts with a group of solid spheres, non-linear aerodynamic behaviors come into effect. The complicated wave reflections such as the Mach reflection occur in the wave propagation process. The wave interactions with vortices behind each sphere's wake cause fluctuation in the pressure profiles of shock waves. This paper reports an experimental study for the aerodynamic processes involved in the interaction between shock waves and solid spheres. A schlieren photography was applied to visualize the various shock waves passing through solid spheres. Pressure measurements were performed along different downstream positions. The experiments were conducted in both rectangular and circular shock tubes. The data with respect to the effect of the sphere array, size, interval distance, incident Mach number, etc., on the shock wave attenuation were obtained.
\end{abstract}

KEY WORDS: shock wave, spheres arrays models, schlieren visualization, pressure measurement, compressible multiphase flow

\section{INTRODUCTION}

The interaction of shock waves with a solid sphere is a fundamental aerodynamic phenomenon ${ }^{[1]}$. The problem of the shock wave interaction with a single particle has been solved by experimental investigations and numerical simulations ${ }^{[2]}$. Britan et al. ${ }^{[3]}$ studied an anti-blast wave structure using a group of small spheres. Purygin and Buzanov ${ }^{[4]}$ reported an application of shock wave impact on solid metal balls, by which the metal surface can be hardened. Though Skews ${ }^{[5]}$ showed a flow visualization of the shock propagation through matrix, the aerodynamics of the shock wave interaction with multiple spheres was not well understood. For example, the experimental data of the shock attenuation by spheres are not sufficient.
The shock interaction with a group of spheres is much more complicated than the interaction with a single sphere because of the nonlinear nature. Firstly, there exist multiple shock wave reflections among spheres; secondly, there exists the shock wave interaction with vortices in the wake behind each sphere. It was because of lack of the aerodynamic knowledge, in their study of particles with diameters from a few micro to a few hundred micro, Rogue et al. ${ }^{[6]}$ did not show clearly how to treat the drag coefficient for the particles in a dense cloud. Sichel et al. ${ }^{[7]}$ also indicated that in the study of shock wave ignition of dusts, both experiments and analyses involve too many difficulties and approximations. In this paper we present an experimental study on the shock interaction with spheres, whose diameters are $10 \mathrm{~mm}$ and $20 \mathrm{~mm}$, respectively, because they can be easily framed by using

Received 2 April 2002, Revised 17 March 2003

* The project supported by the Scientific Research Foundation for the Returned Overseas Chinese Scholars, State Education Ministry, China, and the "BaiRen" Plan of Chinese Academy of Sciences

† E-mail: hhshi@imech.ac.cn 
smaller diameter rods. It is believed that the present experiment may shed some light into the study on particles with smaller diameters.

\section{EXPERIMENTS}

The experiment was done in the $\phi 80$ shock tube ${ }^{[8,9]}$. For the flow visualization, a rectangular section of $730 \mathrm{~mm}$ in length and $55 \mathrm{~mm} \times 53 \mathrm{~mm}$ in cross sectional area is connected to the end of the circular driven section of the shock tube (Fig.1). The total length is increased to $6.12 \mathrm{~m}$. Photos of Figs.2(c) and 2(d) show the structure of the rectangular section.
The test model is shown in Fig.2(a) (front view) and Fig.2(b) (side view), respectively. The frames are constructed with aluminum spheres of $10 \mathrm{~mm}$ and $20 \mathrm{~mm}$ in diameters and stainless rods of $3 \mathrm{~mm}$ in diameter. There are three arrays of particles that are separated at different distances of $10 \mathrm{~mm}, 15 \mathrm{~mm}, 20 \mathrm{~mm}, 25 \mathrm{~mm}$ and $30 \mathrm{~mm}$, respectively. The pressure signals when shock waves passing through the spheres were measured by pressure transducers (Qianfang Type). The positions of the spheres and pressure transducers are shown in Figs.2(c) and 2(d). Sensor 2 is in front of the frame and sensor 1 is behind it. The end wall pressure is

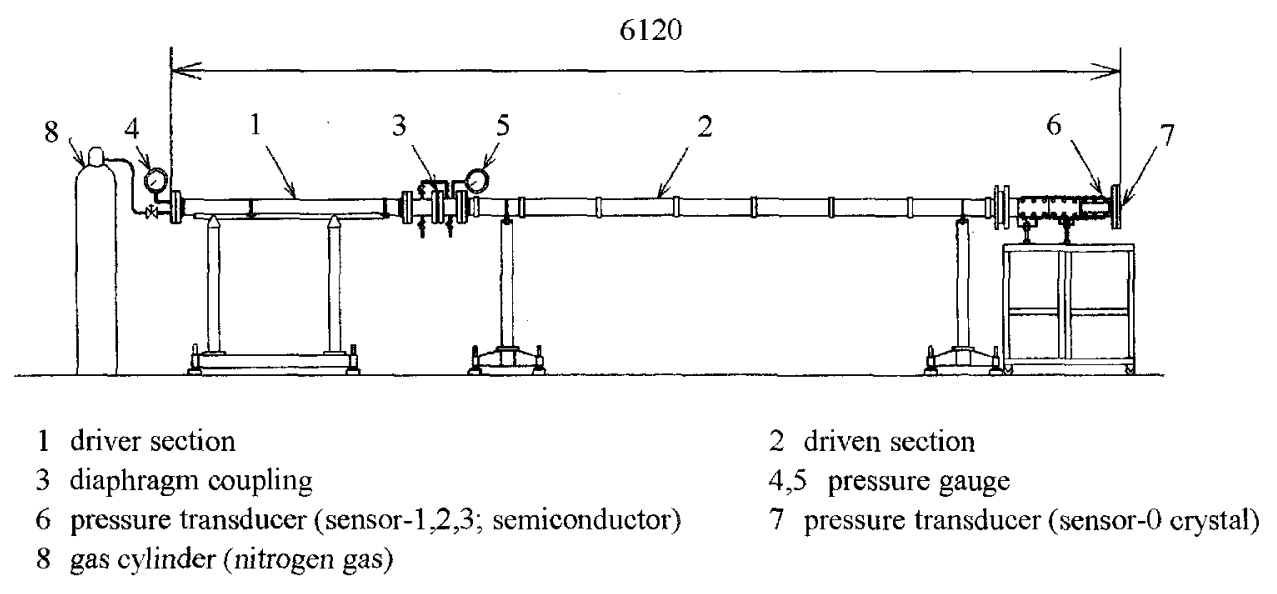

Fig.1 Schematic of the circular/rectangular shock tube

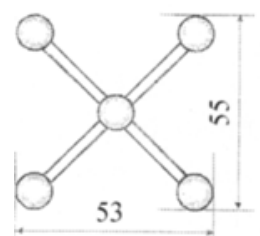

(a)

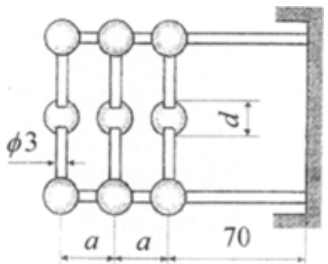

(b)

(c) block-1-103a

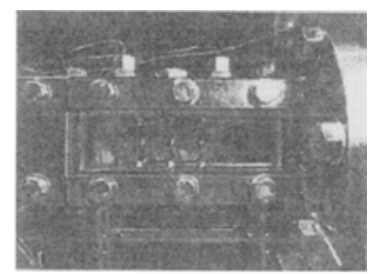

(d) block-1-203a

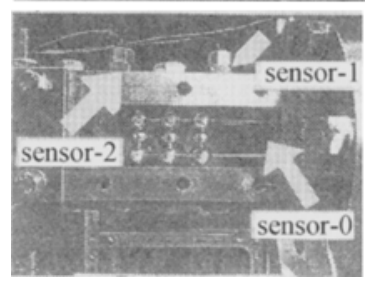

Fig.2 Square frame of solid spheres models 
measured by sensor 0 . The distance between sensors 0 and 1 is $50 \mathrm{~mm}$ and it is $100 \mathrm{~mm}$ between sensors 1 and 2. The reason for choosing the spheres of $10 \mathrm{~mm}$ and $20 \mathrm{~mm}$ in diameter is that they are easily to be machined for constructing the arrays.

In addition to the arrangement of Fig.2, the experiment was also performed in the circular shock tube. The circular frames are made of aluminum spheres of $20 \mathrm{~mm}$ in diameter and stainless rods of $4 \mathrm{~mm}$ in diameter (Fig.3). In Fig.3(a), the frame has three arrays in which two are in $\mathrm{F}$ structure and one is in $\mathrm{R}$ structure. In each array, six spheres are axisymmetrically distributed. The array $R$ is connected to the end wall of the shock tube and it contains no central sphere in order to measure the end wall pressure. In the circular shock tube, the end wall and side wall pressures are measured by transducers $\mathrm{CH} 6$ and $\mathrm{CH} 5$, respectively, which are $92 \mathrm{~mm}$ away ${ }^{[8,9]}$. The frame lengths $l$ and $L$ are variable. Experiments show that the rotation of the frame in the meridian plane (Fig.3(b)) does not influence the pressure measurement. It was also found that the simple sphere structure in Fig.3(c) does not attenuate the shock wave much. Therefore, this paper will only discuss the results of Fig.3(a).
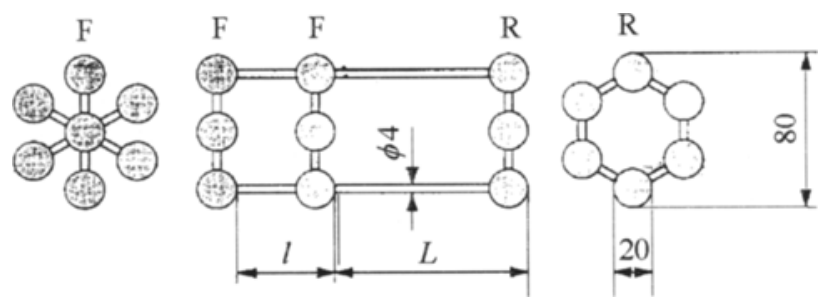

(a) Position A
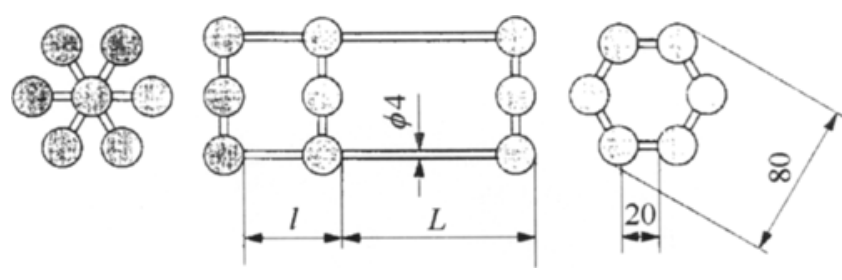

(b) Position $\mathrm{B}$
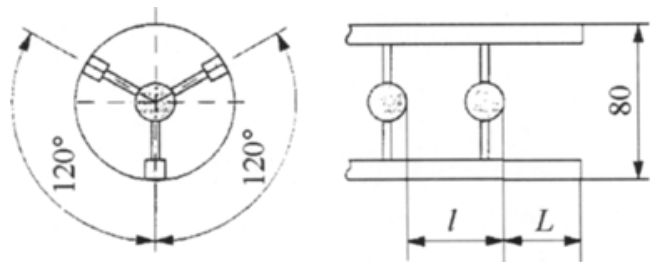

(c) Position $\mathrm{C}$

Fig.3 Circular frame of solid sphere models

A schlieren optical system was used for observing shock wave behaviors. The light source was a pulsed light (Sugahara Laboratory, NP-1A \& NPL-5, $180 \mathrm{~ns}$ exposure time). Two $\phi 150 \mathrm{~mm}$ schlieren mirrors were used. The flash light is triggered by a signal from a pressure transducer located at the entrance of the rectangular section ( $550 \mathrm{~mm}$ from the end wall) when a shock wave passes through it. Figure 4 shows the synchronization system for the flow visualization. Sensor 3 is $400 \mathrm{~mm}$ from sensor 2 and is used to trigger the nano pulse light in the schlieren optical system. The delay time of triggering the nano pulse light can be calculated according to the shock Mach number $M_{s}$. Because the spheres are inserted in the shock tube between sensors 2 and 0 and our main interest is to observe the incident and reflected shock waves 


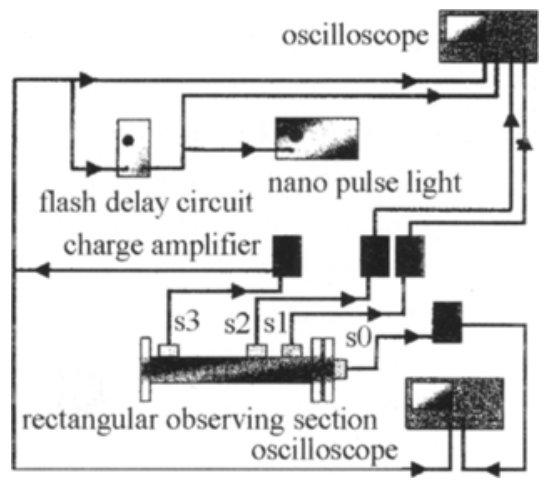

Fig.4 Synchronization system for triggering the nano pulse light in the schlieren optical system

within the distance, with $M_{s}=1.43$, the delay time is about $0.811 \mathrm{~ms} \sim 1.419 \mathrm{~ms}$.

\section{EXPERIMENTAL RESULTS}

\subsection{Wave Behavior}

The process of shock interaction with frame $10320(10 \mathrm{~mm}$ diameter sphere, 3 arrays, $20 \mathrm{~mm}$ interval distance) is shown in Fig.5. In Fig.5(1), the incident shock wave just enters the observation window. In Fig.5(2), the shock wave has passed the first array and meanwhile it is reflected back by the first array. In Fig.5(3), the incident shock is approaching the end wall. It is clear that after passing three arrays of particles, the shape of the wave front has been changed. In Fig.5(4), the incident shock wave is reflected back from the end wall and interacts with the second incident shock wave. This interaction forms a thick reflection shock wave. From Fig.5(3) to Fig.5(6), the turbulent flow is developed from wakes behind spheres and rods.

When the interval distance of the arrays is increased to $25 \mathrm{~mm}$, the wave reflection between two arrays is visible (Fig.6(2)). In Fig.6(3), wavelets are interacting with wakes between the second and the third arrays. Three reflection waves can be seen in Figs.6(2), 6(4) and 6(6), respectively. During shock wave interactions with solid spheres of $20 \mathrm{~mm}$ diameter (frames 20325 and 20330 ), strong wave reflections occur in each array (see Figs.7(2) and 8(2).). After shock wave is reflected back from the end wall, turbulent flows of large scale appear, as shown in Figs.7(4) and $8(4)$. This means that the shock wave is dissipated more quickly. However, these flows do not seem to propagate upstream. This may be due to the fact that the arrays with large spheres block the upstream flow.
(1)

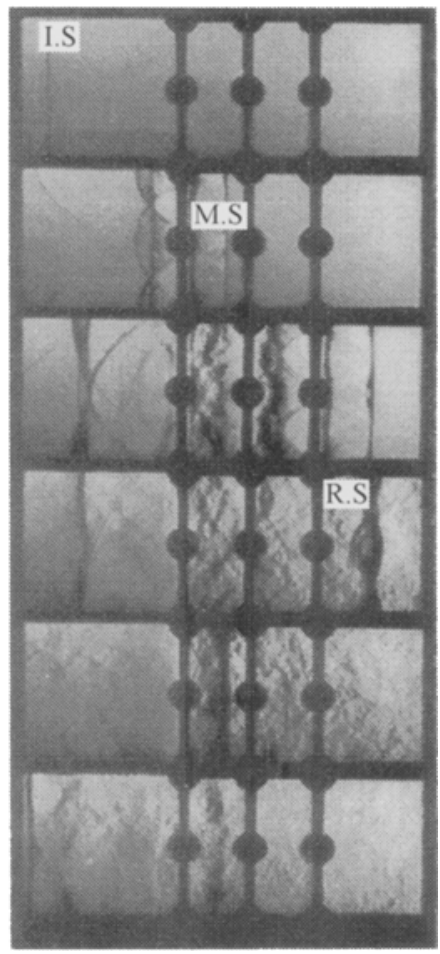

Fig.5 Schlieren photographs of shocksphere interaction. Frame-1$10320, M_{s}=1.43$

(1)

(2)

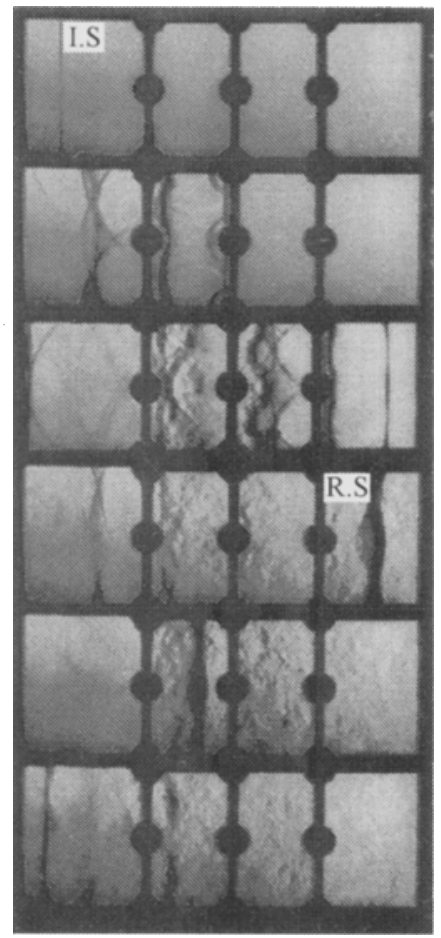

Fig.6 Schlieren photographs of shocksphere interaction. Frame-1$10325, M_{s}=1.43$ 
(1)

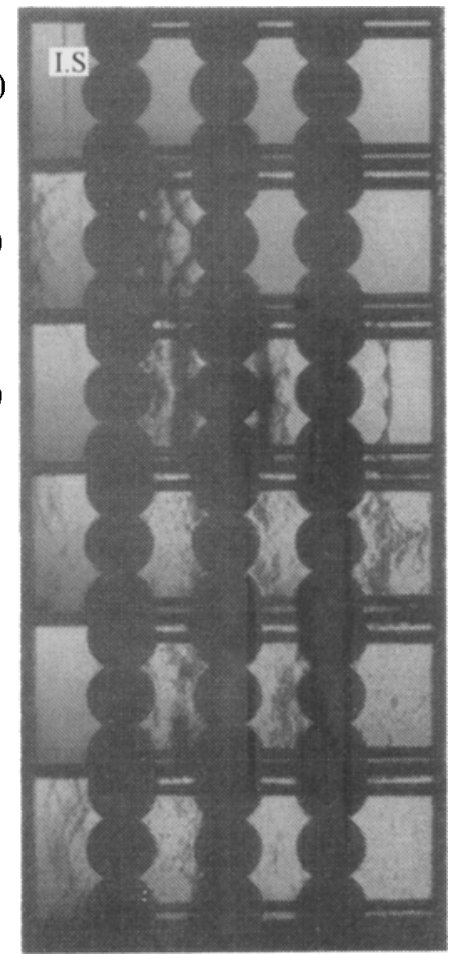

Fig.7 Schlieren photographs of shocksphere interaction. Frame-2$20325, M_{s}=1.43$

(1)

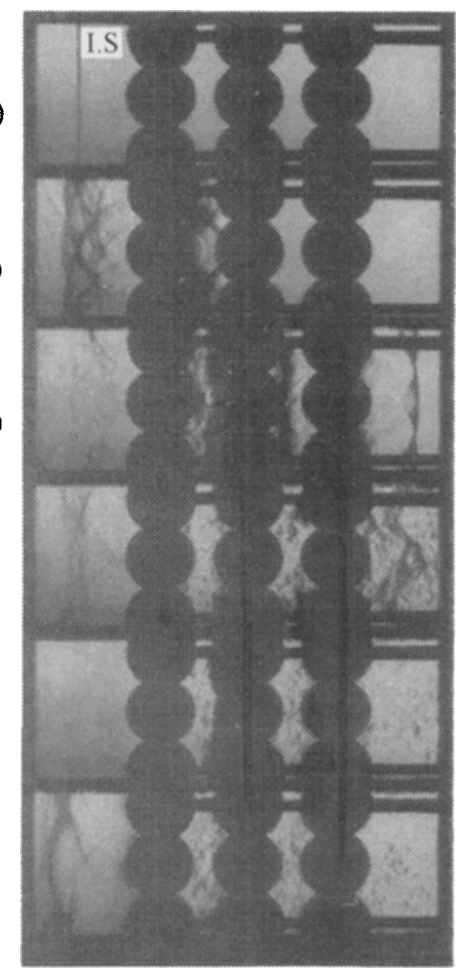

Fig. 8 Schlieren photographs of shockparticle interaction. Frame-2$20330, M_{s}=1.43$
Shock waves are very thin, with a thickness of a few times of the molecular mean free path. For a shock wave $\left(M_{s}=2\right)$ in nitrogen gas in atmosphere at room temperature, the thickness of the shock wave is only about $0.3 \mu \mathrm{m}^{[10]}$. Hence, during the shock wave interaction with solid spheres, once the diameters of spheres are greater than a few micrometers, the wave reflection and diffraction will keep similar. In other words, the phenomena observed in shock/spheres interaction in Figs.5 8 will be seen in the interaction over a wide range of sphere diameter. However, the sphere diameter determines the Reynolds number while a different Reynolds number means the difference of the size of the vortex in the wake of a sphere. Therefore, in the two-phase flow theory, it is necessary to consider the vortex size and turbulent scale into the drag coefficient.

\subsection{Shock Velocity}

The pressure measurement results of frames 10320 and 20330 by sensors 1 and 2 are given in Figs.9(a) 9(d). The theoretical results of shock in air are also shown in the figures (dotted lines). Sensor 2 records the pressure signals of the incident shock wave, the reflected shock wave from the first array
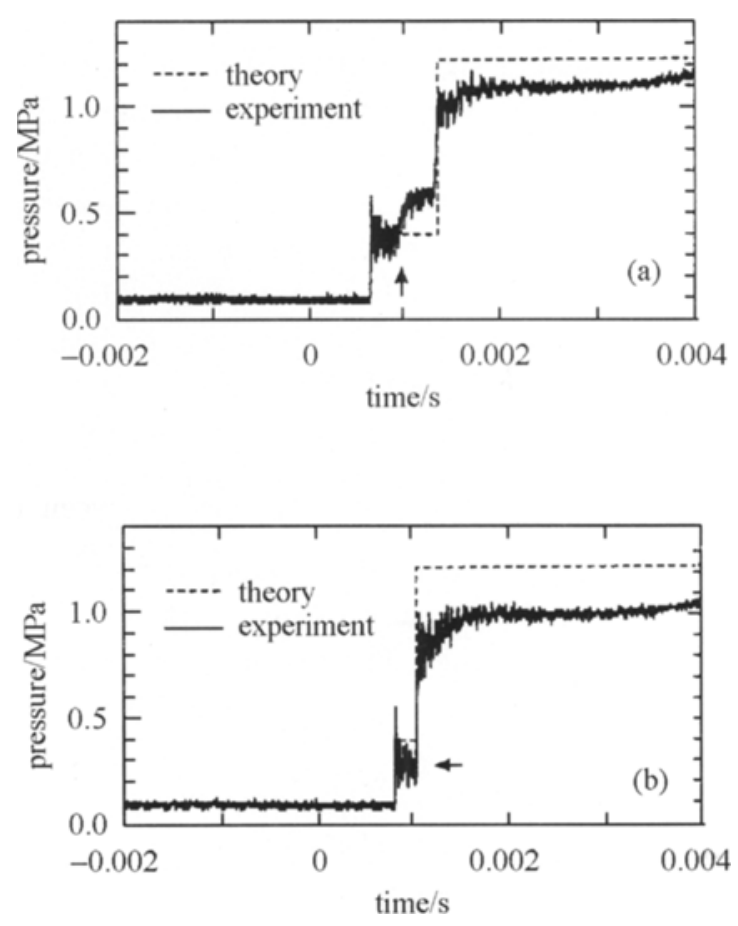

Fig.9 Wave velocity measurement. Incident shock Mach number $M_{s}=1.88$. (a) and (b): $d=10 \mathrm{~mm}, a=20 \mathrm{~mm}$, sensors 2 and 1 ; (c) and (d): $d=20 \mathrm{~mm}$, $a=30 \mathrm{~mm}$, sensors 2 and 1 

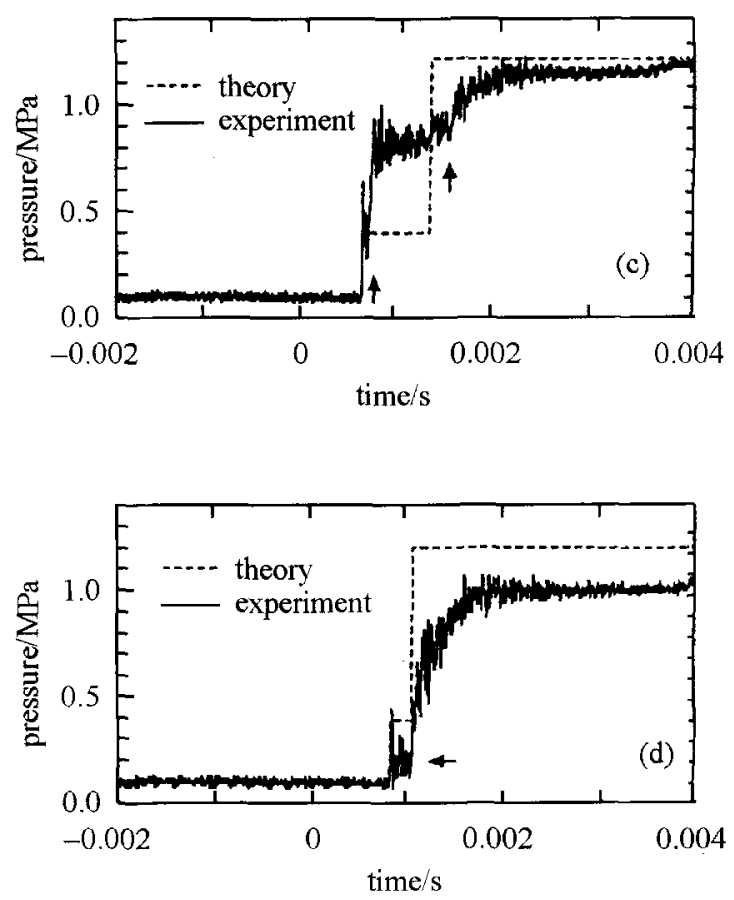

Fig.9 Wave velocity measurement. Incident shock Mach number $M_{s}=1.88$. (a) and (b): $d=10 \mathrm{~mm}, a=20 \mathrm{~mm}$, sensors 2 and 1 ; (c) and (d): $d=20 \mathrm{~mm}$, $a=30 \mathrm{~mm}$, sensors 2 and 1 (continued)

and the transmitted upstream shock wave. Sensor 1 records pressure signals of the transmitted shock wave and reflected shock wave from the end wall. In Fig.9(a), the arrow represents the reflected shock wave from the first array. In Fig.9(b), the arrow represents the pressure $P_{2}$ of the shock wave. From Fig.9(a), it is seen that the measured shock velocity after passing through the particles is in agreement with the velocity in air. From Fig.9(c), it is known that (1) the $20 \mathrm{~mm}$ diameter spheres generate a stronger reflection of shock waves, as is seen in the schlieren photographs (Figs.5 8); (2) the velocity of the shock wave after passing the particles is reduced, which is represented by the slope of the pressure signal (marked by a second arrow on the right). The test mode of $20 \mathrm{~mm}$ diameter spheres occupies $46.5 \%$ of the cross-sectional area in comparison to the $20.3 \%$ area occupation of $10 \mathrm{~mm}$ diameter spheres. This shock velocity reduction may be attributed to the area contraction mechanism as suggested by Monti ${ }^{[11]}$. Furthermore, the present experiment shows that the shock wave velocity reduction begins when the incident Mach number $M_{s}$ is greater than 1.7 .

\subsection{Shock Attenuation}

In all cases, the pressure $P_{2}$ behind the shock wave after the interaction with spheres is less than $P_{20}$ in air (marked by the horizontal arrows in Figs.9(c) and $9(\mathrm{~d})$ ). The ratio $P_{2} / P_{20}$ is defined as the attenuation rate. The results of the test frames of $10 \mathrm{~mm}$ and $20 \mathrm{~mm}$ diameter spheres are given in Figs.10 and 11, respectively. The general tendency is that as the

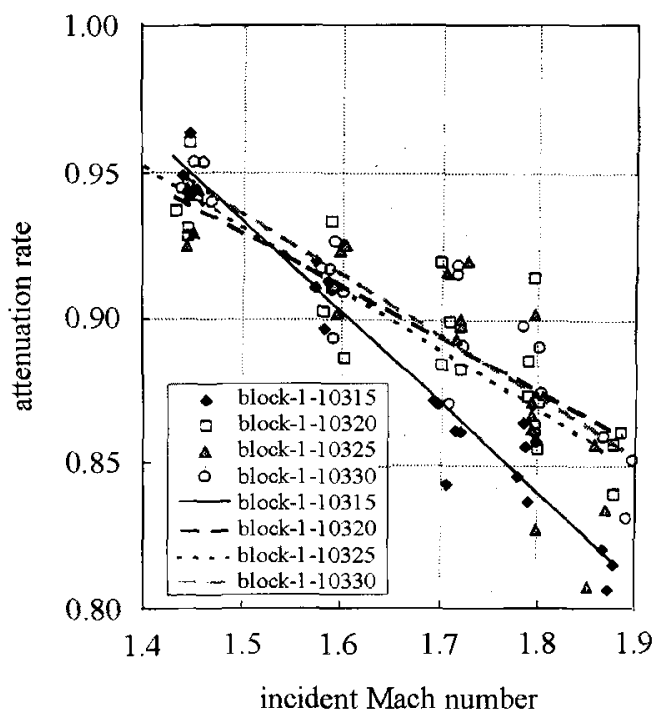

Fig.10 Relationship between the incident Mach number and attenuation rate

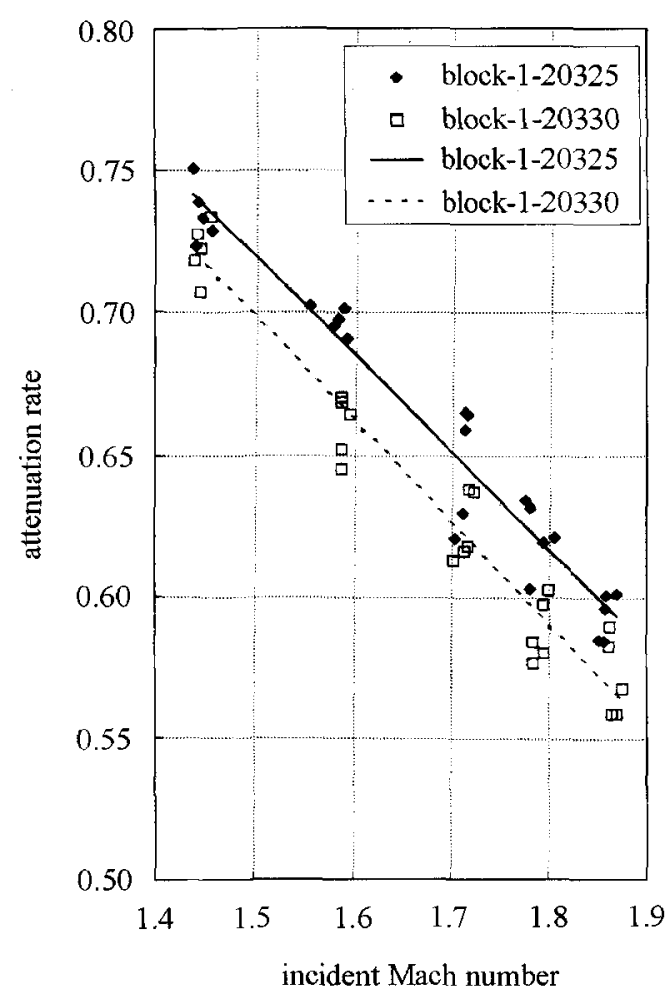

Fig.11 Relationship between the incident Mach number and attenuation rate 
Mach number $M_{s}$ increases, the shock wave is more attenuated. Qualitatively, for the $20 \mathrm{~mm}$ diameter spheres, a greater attenuation occurs in the spheres with a larger interval distance. For the $10 \mathrm{~mm}$ diameter sphere, if the interval distance is larger than $15 \mathrm{~mm}$, this effect does not occur. That is, Fig. 10 shows that the attenuation rate of the 10315 frame is the biggest whereas the attenuation rates for the other three frames are quite close.

The results from the circular shock tube are given in Fig.12. Here the shock attenuation variation is represented by the ratio of $P_{2} / P_{1}$ against $P_{4} / P_{1}$. The different length $L$ of the particles (see Fig.3) causes different attenuation rates. The solid circles represent the measurement results in air. The solid line is the theoretical result. In case of $L=200 \mathrm{~mm}$, it is clear as the sphere number increases, the shock is more attenuated. In case of $L=100 \mathrm{~mm}$, although the shock is attenuated, the sphere number does not play a role. This may be due to the fact that the pressure transducer $\mathrm{CH} 5$ was too close to the solid spheres because there was only $9 \mathrm{~mm}$ gap between them.

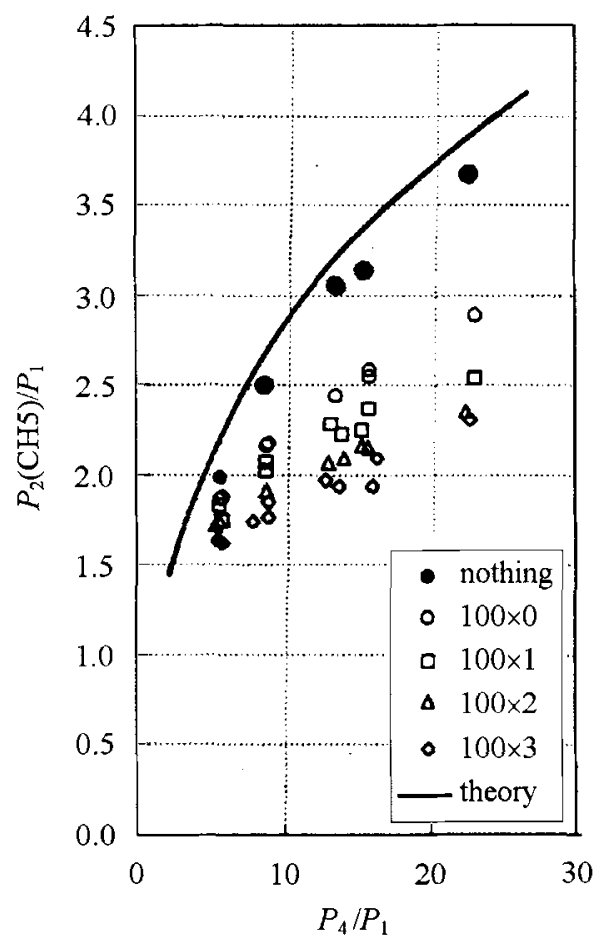

Fig.12 Effect of sphere array number on shock pressure $P_{2}$ at $\mathrm{CH} 5$ (position A). $L=200 \mathrm{~mm}$

\subsection{Pressure Multiplying}

In a circular shock tube, if solid spheres near the end wall are axisymmetrically distributed, a phenomenon called the pressure multiplying near the end wall may occur. In the test model shown in Fig.3(a), the array $R$ is such a structure. Figure 13(b) shows the measured pressure multiplying, i.e., the peak pressure $P_{\max }$ reaches almost $0.6 \mathrm{MPa}$ whereas the end wall pressure in air $P_{5}$ is measured as $0.4 \mathrm{MPa}$ (Figs.13(a) and 13(b)). The pressure multiplying brings about a pressure increase of 1.5 times. Of course, if the shock wave has been largely attenuated before impact on the last array of spheres, the pressure multiplying may not occur. The experiments with the spheres models of $l=50 \times n$ and $100 \times n(n=0,1,2,3) \mathrm{mm}$ and $L=100 \mathrm{~mm}$ and $200 \mathrm{~mm}$ show that the pressure multiplying becomes effective when the array $R$ has one or two layers. Figure 14 is a typical result. When the array $R$ has three or four layers, the pressure multiplying cannot compensate the pressure loss by the shock attenuation. In the case of $n=0$, it is found that the peak pressure $P_{\max }$ always appears at a different shock Mach number from 1.4 to $1.9^{[12]}$. Considering that this phenomenon does not occur in the rectangular shock tube, it is thought that the pressure multiplying is due to shock focusing. Of course, it is worth while to carry out further flow visualizations to show that the shock focusing indeed occurs near the end wall region.

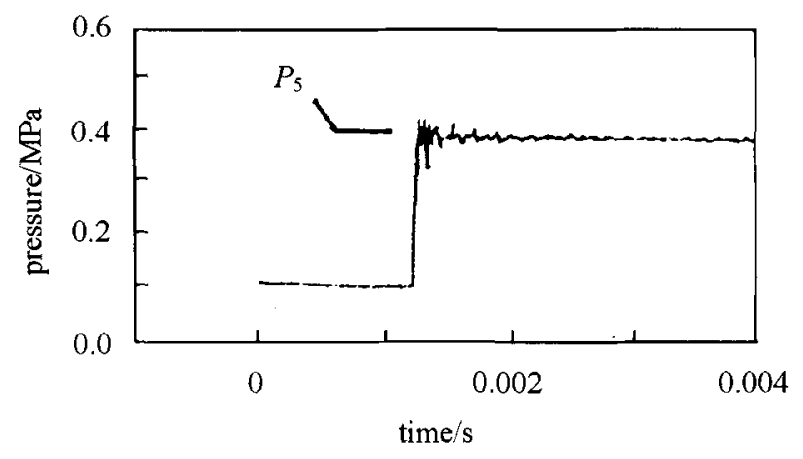

(a) Empty in the tube

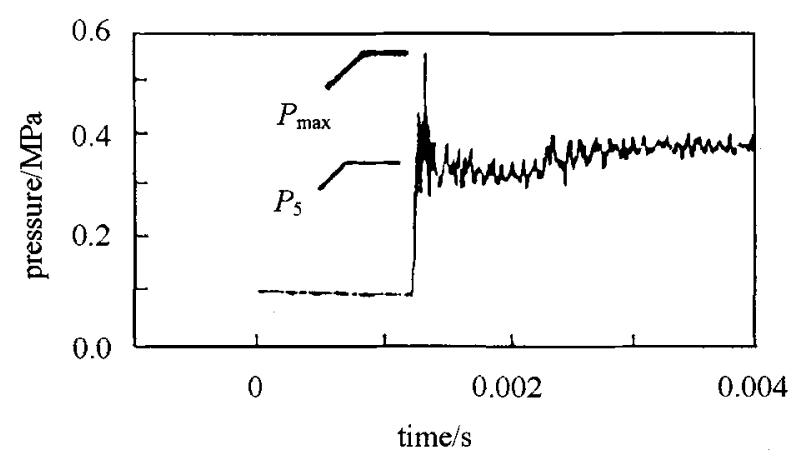

(b) $L=200 \mathrm{~mm}, n=0$

Fig.13 Pressure multiplying in the circular shock tube. Incident shock Mach number $M_{s}=1.4$ 


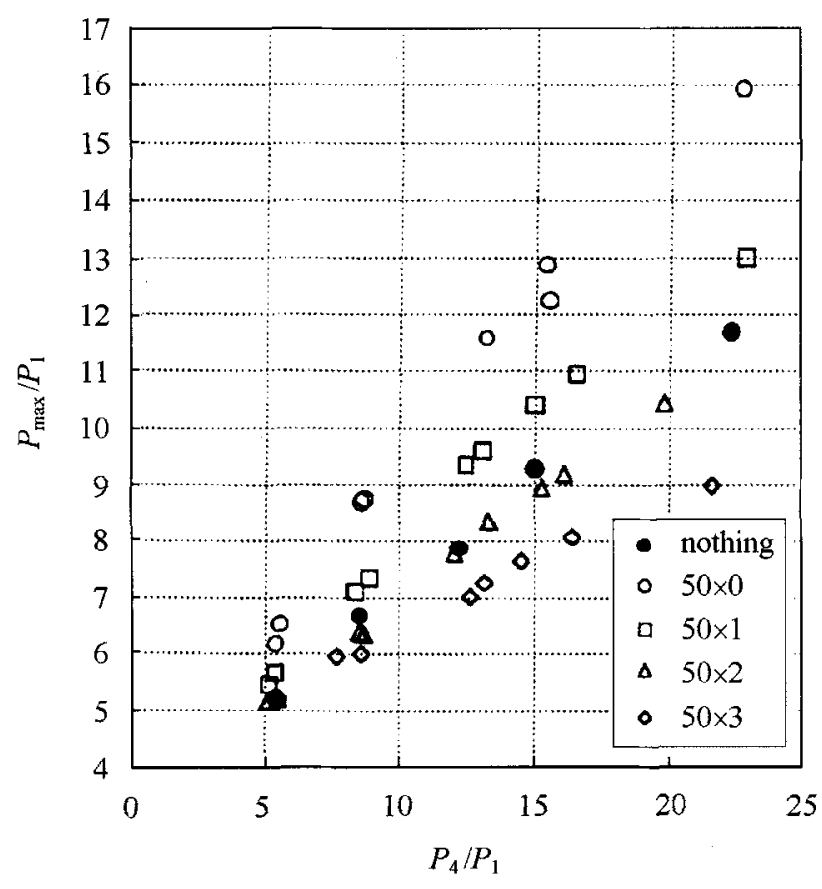

Fig.14 Peak pressure of reflected shock wave at $\mathrm{CH} 6 . \quad l=50 \times n(n=0$, $1,2,3) \mathrm{mm}, L=200 \mathrm{~mm}$

\subsection{End Wall Pressure}

Figure 15 gives the end wall pressure $P_{5}$ in the rectangular shock tube. The results reveal that the
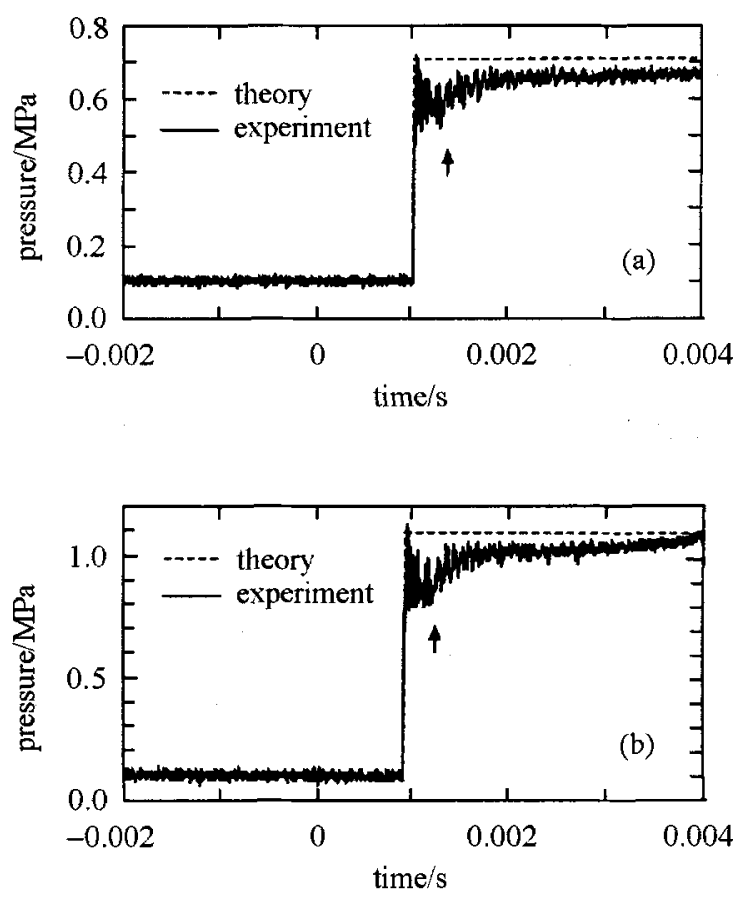

Fig.15 End wall pressures. Sensor 0. (a) and (b): $d=10 \mathrm{~mm}, a=20 \mathrm{~mm}$, $M_{s}=1.6$ and $1.8 ;(c)$ and (d): $d=20 \mathrm{~mm}, a=30 \mathrm{~mm}, M_{s}=1.6$ and 1.8
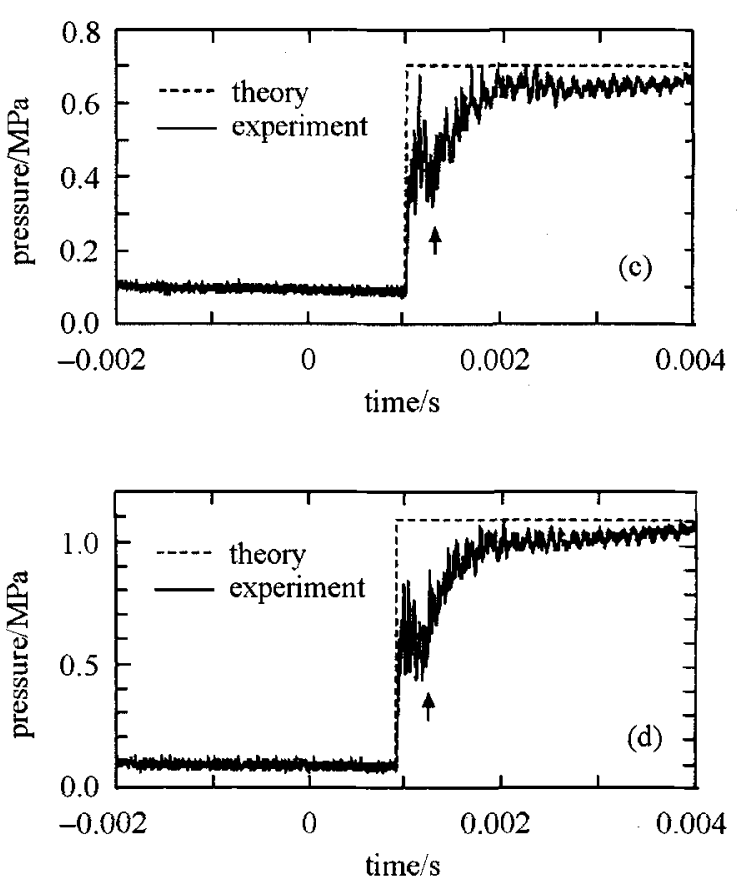

Fig.15 End wall pressures. Sensor 0. (a) and (b): $d=10 \mathrm{~mm}, a=20 \mathrm{~mm}$, $M_{s}=1.6$ and $1.8 ;$ (c) and (d): $d=20 \mathrm{~mm}, a=30 \mathrm{~mm}, M_{s}=1.6$ and 1.8 (continued)

shock waves have complicated structures after passing a solid sphere. As seen in the measurement results of $P_{2}$ for the shock attenuation, the solid spheres with $20 \mathrm{~mm}$ diameters block the way for the shock wave to reach the end wall. The pressures in Figs.15(c) and $15(\mathrm{~d})$ mean that the shock waves have been dissipated before reaching the end wall. These results are in agreement with the optical observation shown in Figs.5 8. The effect of the sphere array number on the end wall pressures in the circular shock tube has been studied with the particle models of $l=100 \times n(n=0,1,2,3) \mathrm{mm}$ and $L=100 \mathrm{~mm}$ and $200 \mathrm{~mm}$. The pressure reduction can be quantitatively found by plotting the results into the relationship of $P_{5} / P_{1}$ against $P_{4} / P_{1}$. It is found that as the array number $n$ increases, the end wall pressure is decreased. If $P_{4} / P_{1}=22.5$, the theoretical value of $P_{5} / P_{1}$ is 12 whereas it becomes $4.4 \sim 7.7$ when the spheres are present.

\section{CONCLUSIONS}

(1) When a shock wave passes solid spheres, the shock wave is attenuated. The shock attenuation largely depends on the sphere number, the sphere diameter and the spatial distribution as well as the incident Mach number. The multiple shock reflections 
among spheres and the shock interaction with vortices behind the spheres cause a complicated shock structure and pressure signal. The rarefaction wave in the shock wave interaction with small particles suggested by Boiko et al. ${ }^{[13]}$ has not been found. The reasons for this may be that (1) the sphere models used in this experiment are stationary; (2) in the mathematical model of Boiko et al., they assume that there is an interface between the gas and the two-phase mixture, which will produce a solution of the expansion wave.

(2) Within the scope of the present experiment, the reduction of the shock wave velocity only occurs when the area contraction and the incident Mach number are large. The shock velocity in the spheres of $10 \mathrm{~mm}$ in diameter does not change. The shock velocity for the spheres of $20 \mathrm{~mm}$ in diameter changes significantly when $M_{s}$ is greater than 1.7.

(3) The pressure multiplying may occur in the shock/ spheres interaction. This phenomenon is one of the key issues in the nonlinear aerodynamics of shock/sphere interactions.

\section{REFERENCES}

1 Shapiro AH. The Dynamics and Thermodynamics of Compressible Fluid Flow, Vol II. New York: The Ronald Press Company, 1954. 880 888

2 Takayama K. Itoh K. Unsteady drag over circular cylinders and aerofoils in transonic shock tube flows. Rep Inst High Speed Mech, Tohoku University, Japan,
1986, 51: 1 41

3 Britan A, Ben-Dor G, Igra O, et al. Shock waves attenuation by granular filters. Int $J$ Multiphase Flow, 2001, 27: 617 634

4 Purygin NP, Buzanov VI. Quasi-spherical pulsed loading impact on solid metal balls. In: Proc 19th Int Symp on Shock Waves, Vol III, Marseille, France, 2630 July, 1993. 307 310

5 Skews BW. Shock wave impact on porous materials. In: Proc 19th Int Symp on Shock Waves, Vol III, Marseille, France, 26 30 July, 1993. 11 20

6 Rogue X, Rodriguez G, Haas JF, et al. Experimental and numerical investigation of the shock-induced fluidization of a particles bed. Shock Waves, 1998, 8: $29 \sim 45$

7 Sichel M, Baek SW, Kauffman CW, et al. The shock wave ignition of dusts. AIAA J, 1985, 23: 1374 1380

8 Shi HH, Kawai K, Itoh M. Attenuation and reflection of shock waves in low density foams in a shock tube. AIAA 99-0143, 1999

9 Shi HH, Kawai K, Itoh M, et al. The interaction between shock waves and foam in a shock tube. Acta Mechanica Sinica, 2002, 18: 288 301

10 Ikui T, Matsuo K. Mechanics of Shock Wave. Tokyo: Korona Press, 1983. 58 60 (in Japanese)

11 Monti R. Normal shock wave reflection on deformable walls. Meccanica, 1970, 4: 285 296

$12 \mathrm{Shi} \mathrm{HH}$. Using shock tube to investigate supersonic gas-solid two-phase flow. Chinese J Appl Mech, 2003, 20(4): $41 \sim 45$ (in Chinese)

13 Boiko VM, Kiselev VP, Kiselev SP, et al. Shock wave interaction with a cloud of particles. Shock Waves, 1997, 7: 275 85 